\title{
Pratiques
}

Linguistique, littérature, didactique

\section{Positions énonciatives pour apprendre dans les différentes disciplines scolaires : une question pour la didactique du français?}

\section{Martine Jaubert et Maryse Rebière}

\section{OpenEdition}

\section{Journals}

Édition électronique

URL : http://journals.openedition.org/pratiques/1718

DOI : $10.4000 /$ pratiques. 1718

ISSN : 2425-2042

Éditeur

Centre de recherche sur les médiations (CREM)

Édition imprimée

Date de publication : 15 juin 2011

Pagination : 112-128

Référence électronique

Martine Jaubert et Maryse Rebière, «Positions énonciatives pour apprendre dans les différentes disciplines scolaires : une question pour la didactique du français ? », Pratiques [En ligne], 149-150 | 2011, mis en ligne le 17 juin 2014, consulté le 01 mai 2019. URL : http:// journals.openedition.org/pratiques/1718; DOI : 10.4000/pratiques. 1718 


\title{
Positions énonciatives pour apprendre dans les différentes disciplines scolaires : une question pour la didactique du français ?
}

\author{
Martine Jaubert \\ Maryse Rebière \\ IUFM-Bordeaux 4, LACES, Université de Bordeaux
}

\section{Introduction}

La question du langage pour apprendre est de celles qui interpellent la discipline français dans ce qui fait son identité historique. D'une part, personne ne remet en cause la nécessité d'une discipline consacrée à apprendre à lire, écrire, parler, réfléchir sur le fonctionnement des textes et du langage ; d'autre part, depuis de nombreuses années, il semble relativement admis que les savoirs et les compétences qui fondent cette discipline contribuent à la construction des connaissances propres à l'ensemble des disciplines scolaires. Dès lors se pose la question de l'articulation entre cette discipline et les autres : le lire, l'écrire, le parler, la réflexion sur le langage seraient-ils indépendants des contextes d'utilisation, et s'actualiseraient-ils d'eux-mêmes, " naturellement», dans l'ensemble des situations où le langage est à l'œuvre ? La discipline " français » serait alors fermée sur elle-même. D'un autre côté, ne risque-t-elle pas de se dissoudre dans les contextes d'utilisation, en histoire, en mathématiques, en toutes disciplines dont la littérature, pour se réduire à l'apprentissage de quelques mécanismes morpho-syntaxiques? L'identité culturelle historique de la discipline serait-elle menacée par sa propre extension? Cette contribution se propose ainsi d'explorer la question de la place du langage pour apprendre dans les disciplines scolaires et de sa prise en charge par la discipline du français. Dans la zone de contact entre le champ de la didactique du français et celui des didactiques des disciplines autres, le fonctionnement des langages est un objet qui reste à la marge de la didactique du français. Or il est aussi à la marge des didactiques des autres disciplines, 
comme si la maîtrise du langage allait de soi et ne relevait pas d'apprentissages spécifiques, comme si le langage servait simplement à coder une pensée préconstruite et comme si les savoirs existaient en soi hors des pratiques, dont les pratiques langagières qui les ont façonnés. Cependant, depuis une dizaine d'années, les travaux de recherche sur ce sujet, les colloques et ouvrages souvent collectifs et pluridisciplinaires se multiplient.

A partir de certains de ces travaux, cette contribution interrogera, autour de la notion de « position énonciative », à la fois les fondements épistémologiques du rôle accordé au langage dans les différentes disciplines, mais aussi la configuration «classique » de la didactique du français, certains de ses objets, ses points aveugles « que le français exclut de son champ faute de place ou de reconfiguration » (Halté, 1998/2008, p. 36) ainsi que la nécessité de prendre en compte de nouveaux objets d'enseignement « univers de discours, sémiotique spécifique, métalangages disciplinaires, spécification de conduites langagières »(idem). En effet, un des enjeux majeurs de cette question consiste à repenser l'enseignement de l'activité langagière, les rapports que le système linguistique entretient avec la réalité extralinguistique, la sphère d'échanges. A la lecture de ces travaux, nous faisons l'hypothèse qu'un des objets au centre de cette problématique qui permettrait de reconfigurer l'enseignement du français, comme le prône Bernard Combettes, (2010, p. 15) et d'articuler langage et cognition, pourrait être la notion de « position énonciative » qui caractérise selon nous les différentes activités langagières disciplinaires. Que prévoient les curriculums pour permettre à tous les élèves de construire les positions énonciatives pertinentes et de s'approprier les pratiques langagières attendues en chaque discipline ? Quelles théories et approches du langage privilégier dans cette optique ? Il est, selon nous, de la responsabilité de la didactique du français de réfléchir aux conditions permettant aux élèves de prendre conscience de ces variations et de ces jeux de langage et d'outiller les enseignants sur les activités langagières pour apprendre dans les disciplines.

\section{1. Émergence d'un objet d'investigation dans le champ des didactiques disciplinaires}

\subsection{Le langage pour apprendre dans les disciplines : un objet scolaire qui cherche un ancrage disciplinaire ${ }^{(1)}$}

Le lien entre langage et disciplines n'est pas nouveau dans les instructions officielles depuis Jules Ferry, particulièrement en ce qui concerne l'enseignement des sciences à l'école ( $c f$. la leçon de choses et la pédagogie de l'éveil), quelles que soient les fonctions attribuées au langage (Schneeberger et Vérin, 2009, 4647) qui, avec la géographie, entretient un rapport au réel particulier. C'est pourquoi jusque dans les années soixante-dix, le langage est considéré comme un système de codage du réel : il est pensé en termes de langue et non d'activité langagière. Des recherches de l'INRP (G. Ducancel et V. Host) qui explorent la na-

(1) Référence à la distinction opérée par Daunay et Denizot, Pratiques 133-134, 2007. 
ture des liens entre langage et apprentissages scientifiques (V. Host, 1983), et plus largement la question de la transversalité du langage et de sa place dans les apprentissages disciplinaires, infléchissent ce point de vue et initient des travaux publiés dans une revue scientifique (Aster 4, 1987 et 6, 1988).

Suite à la massification des collèges (années 70) et dans le mouvement du Plan Rouchette, une responsabilité dans la réussite scolaire est attribuée à la langue en toutes disciplines et la discipline français devient «compétence transversale » avec les directives ministérielles de 1989 (la maîtrise de la langue à l'école, 1992, au collège, 1997). Le double statut d'objet d'étude et de moyen est ainsi reconnu au langage, bien que les textes restent fidèles au vocable « langue ». Cependant les textes de cette période hésitent dans l'ancrage disciplinaire de ce qui est résolument un objet scolaire. Ni compétences transversales, ni compétences disciplinaires, les compétences dans le domaine de la langue se voient attribuer un chapitre (1995, pp. 93-104) et donc un statut spécifique. D’objet scolaire, le « langage pour apprendre » devient objet enseignable en tant que tel, dans La maîtrise de la langue à l'école (1992) et dans les IO de 1995. Les textes postulent la nécessité de construire une langue transdisciplinaire, hors des différentes disciplines $(1995$, p. 105). Cet objet trouve " naturellement » sa place dans le champ des recherches en didactique du français qui, nourrie des travaux sur les typologies, les discours, l'énonciation et des questions de rhétorique et de pragmatique, etc., travaille à l'identification, au fonctionnement et aux spécificités linguistiques des types de texte puis des genres de discours, mais indépendamment de leur contexte d'utilisation : description, explication, argumentation, consignes... comme en attestent les titres de la revue Pratiques, par exemple, depuis 1980 (Argumenter, 1980 ; L'argumentation écrite, 1992 ; Enseigner l'argumentation, 1997 ; Les textes explicatifs, 1986 ; Les discours explicatifs, 1988...). La même remarque peut être faite pour la revue Repères (12, 1995 et 17, 1998).

Ce point de vue va progressivement évoluer dans les textes, de La maîtrise de la langue au collège (1997) qui prône la prise en charge par les disciplines de leurs spécificités langagières, hors d'un "illusoire noyau transdisciplinaire » (1997, p. 39), jusqu'aux textes officiels pour l'école de 2002 qui donnent à chaque discipline une responsabilité dans la construction des langages disciplinaires, se positionnent clairement pour la maitrise « des discours» (2002, 173-176) et listent pour chacune des objectifs langagiers. Tout se passe comme si la «maitrise de la langue » était tributaire de la relation entre les objets disciplinaires, les modes d'apprendre propres à chaque discipline et les pratiques langagières qui donnent leur substance à ces objets et à ces modes d'apprendre.

La question se pose alors des objets disciplinaires du français - qui ne peuvent plus se réduire à la langue et à la littérature - et de la cohérence d'ensemble de la discipline menacée d'implosion, question centrale du colloque de l'AIRDF (2) (anciennement DFLM) «Le français, discipline singulière, plurielle ou transversale », Québec, 2004.

(2) DFLM : Didactique du Français Langue Maternelle ; AIRDF : Association Internationale pour la Recherche en Didactique du Français. 


\subsection{Un objet théorique?}

En effet, on constate l'apparition, depuis une dizaine d'années, de travaux de recherche portant sur le langage dans les différentes disciplines, que leurs auteurs s'inscrivent dans les sciences du langage ou dans une ou plusieurs disciplines spécifiques (EPS, histoire, SVT... mais aussi étude de la langue et littérature). Notre contribution ne fera état que de quelques-unes de ces recherches. Elle ne vise pas l'exhaustivité et peut passer sous silence des travaux importants. Il s'agit seulement ici de tenter de dessiner un parcours, des tendances de la recherche.

\subsubsection{Un objet dissonant, difficile à construire...}

Au début des années 2000, les discours des didacticiens qui s'intéressent au langage dans leur discipline, convergent vers une représentation du langage qui ne se réduit pas à un code transparent, sans pour autant expliciter systématiquement leur ancrage théorique.

Pour exemple, La Lettre de la DFLM, n²6 2000-1, qui présente un dossier "Ecrire pour apprendre », donne la parole à des chercheurs de champs autres (Audigier, F., Histoire-géographie et Gemmene, L., Lejeusne, M., Leroy, A., Romainville, M., sciences). Si les formulations établissent des liens étroits entre langage et pensée, attribuent à l'écrit un « rôle heuristique», parlent d'écrit « miroir » (au sens de réflexif), « scalpel » de la pensée, « laboratoire » de construction des connaissances et de « formulations... empreintes de son cerveau [de l'élève] », en revanche rares sont les théories linguistiques convoquées et les quelques références à Saussure et à Benveniste qui apparaissent dans certaines bibliographies (F. Pluvinage, 2000, pour les mathématiques et J.-C. Marchand, 2002 , pour la musique) ne signalent pas la dimension sociale, constitutive pourtant de leur théorie mais occultée par la lecture traditionnelle.

Au contraire, dans le même numéro, des didacticiens du français (BarréDe Miniac, C., Bucheton. D., Chabanne, J.-C., Reuter, Y.) développent des entrées théoriques en référence à Goody (écrit considéré comme « opérateur cognitif », constructeur de nouveaux rapports aux savoirs et aux langages), à la perspective culturelle portée par les travaux autour de la littéracie (questions des différentes modalités d'accès à l'écrit et de leurs conséquences dans le cadre scolaire, rapports au langage et à l'écrit). Ces didacticiens, via les travaux d'anthropologues, de sociologues et socio-linguistes considèrent les discours comme des construits sociaux, s'intéressent aux postures des élèves et signalent un nouvel objet inscrit dans la perspective socio-historique : les écrits réflexifs. Cependant s'ils s'interrogent sur le rapport au langage construit socialement par les élèves, la question de la spécificité disciplinaire de ce rapport au langage n'est pas évoquée.

Cette partition constatée dans l'organisation de la revue, tout à fait compréhensible, pose un des problèmes clés de l'objet « pratiques langagières dans les disciplines » : peut-on parler des pratiques langagières quelle que soit la discipline, en ignorant les savoirs, les pratiques, les modes de penser-parler-agir de chacune et inversement, peut-on parler de pratiques langagières disciplinaires 
en ignorant le fonctionnement du langage, les théories qui le décrivent et les modes de penser-parler-agir de la discipline dont l'objet est le langage ? ${ }^{(3)}$

\subsection{2. ...en cours d'orchestration ${ }^{(4)}$}

Cette dernière décennie, les travaux de recherche pour mieux appréhender la question des pratiques langagières se diversifient et se multiplient au sein des disciplines. Plusieurs colloques s'intéressent à la question et permettent des échanges de cadres théoriques et de pratiques, contribuant ainsi à construire l'objet « langage pour apprendre » sur le plan épistémique et plus largement didactique ( $c f$. Bordeaux 2003, Construction des connaissances et langage dans les disciplines d'enseignement; Lille 2004 Faut-il parler pour apprendre?; Québec, 2004, Le français, discipline singulière, plurielle ou transversale ; Bordeaux, 2005, Les références épistémologiques ; Bordeaux, 2008 Didactiques, quelles références épistémologiques?...)

Les sciences, historiquement sensibles à la question du langage, sont un terrain d'étude privilégié pour analyser les relations langage et conceptualisation, pour les chercheurs en didactique des sciences comme en didactique du français dont les articles sont croisés dans les publications et parfois co-signés par des chercheurs des deux champs (Aster 33, Argumenter en classe de sciences, C. Buty et C. Plantin, 2008, Développer des pratiques d'oral et d'écrit en sciences, P. Schneeberger et A. Vérin, 2009, Pratiques 143-144, Ecrits de savoirs). I1 n'est donc pas étonnant que de nombreuses communications lors des colloques mentionnés ci-dessus concernent le langage en sciences (Bordeaux, 2003, une trentaine de communications dont le titre réfère aux disciplines scientifiques ${ }^{(5)}$; Lille, 2004, 17 communications, Québec, 2004, 6 communications). Cette orientation de l' INRP s'est étendue à d'autres disciplines comme en témoignent par exemple Argumentation et disciplines scolaires (J. Douaire, 2004), Apprendre à écrire au collège dans les différentes disciplines (C. Barré-De Miniac et Y. Reuter, 2006).

D'autre part, on commence à observer des co-directions de thèse associant didacticien d'une discipline et didacticien du français (thèses de Yann Lhoste, 2008, d'Aurélie Dupré, 2010), des jurys mixtes de thèse (Marlot, 2008, SVT, Doussot, 2009, Histoire, Quilio, 2009, mathématiques, Dupré, 2010, SVT, Saïd, 2010, SVT). On peut aussi s'interroger sur la portée, pour la question qui nous concerne, de la présence d'une psychologue (F. Wynnikamen) qui s'intéresse à

(3) Il est notable que pendant cette décennie, les didacticiens du français qui travaillaient sur le rôle des pratiques langagières dans la conceptualisation, par exemple en sciences, étaient considérés par leurs pairs comme des didacticiens des sciences, alors que les didacticiens des sciences qui analysaient les pratiques langagières de leur discipline avec des outils empruntés aux sciences du langage, n’étaient plus considérés par leurs pairs comme des scientifiques...

(4) Référence incontournable à Volochinov-Bakhtine, l'ancrage social des discours, le dialogisme source d'hétéroglossie.

(5) Ce colloque est également marqué par l'analyse de l'activité langagière spécifique dans les sous-disciplines du français, grammaire et littérature. Et il faut aussi signaler une recension reflétant l'essor de la démarche comparatiste : voir la communication de M.-L. Schubauer-Leoni, « La fonction des dimensions langagières dans l'évolution d'un ensemble de travaux sur le contrat didactique ». 
la métacognition dans le jury d'une thèse consacrée aux interactions en EPS (Darnis, 2004). En outre, des travaux à visée comparatiste commencent à faire 1'objet de thèses (Bedoin, 2008, littérature et sciences, Dupré, 2010, pratiques de lecture en étude de la langue et en sciences).

\section{Cadrage de l'objet}

\subsection{Cadrage théorique des recherches dans les différentes disciplines}

Si nous prenons l'exemple de quelques thèses récentes s'intéressant au langage dans les disciplines, nous constatons un traitement très différencié, sous l'angle théorique, de la question langagière.

Certaines thèses privilégient les théories interactionnistes. Le langage y est principalement appréhendé dans un contexte socio-constructiviste visant la construction de compétences dans l'interaction sociale. Les interactions entre élèves sont étudiées comme moyen de la conceptualisation, dans le cadre par exemple du tutorat entre élèves (Caillier, 2005), ou comme support d'une analyse (pénétrante, bien que plus pédagogique que didactique) des divers univers dans lesquels les élèves sont appelés à circuler dans le cadre de « récits éducatifs » (Decron, 2007). Dans d'autres cas, si le processus de conceptualisation est observé, c'est pour mieux identifier les facteurs qui pèsent sur ces interactions : dominant/dominé, garçon/fille, formes de regroupement (dyades, groupes... homogènes/hétérogènes) (Darnis, 2003, EPS, discipline nourrie par les recherches nord américaines sur l'apprentissage coopératif).

Au-delà de ces exemples, le colloque de Lyon (2010) sur les interactions didactiques a mis en évidence l'importance des interactions dans les recherches en didactique et la fragilité de la dimension didactique, « réduite à un décor » (Bernié, grand témoin), ce que met aussi en cause Elisabeth Bautier (Pratiques, 2010, 18 19) lorsqu' elle conteste 1' " affaiblissement du discours instructeur (Bernstein, 2007) au profit des échanges entre élèves et entre l'enseignant et les élèves ». La focalisation sur les interactions déplace le regard des dispositifs didactiques vers les échanges, et des apprentissages disciplinaires vers des apprentissages communicationnels. Par ailleurs, « on y retrouve une conception de la langue, instrument de communication, pensée comme transparente, d'accès partagé par tous » (Bautier, idem).

En Sciences et en Histoire, certains chercheurs, qu'ils soient linguistes (équipe de Bordeaux, Bernié, 2001, Jaubert, 2001, 2007, Rebière, 2001) ou spécialistes de l'une ou l'autre de ces disciplines (Schneeberger, 2005, Lhoste, Y, 2007, Doussot, 2009, Kessas, thèse en cours), convoquent les Pratiques Sociales de Référence (introduites dans le champ de la didactique par Martinand, 1981) des sphères de production des savoirs. Ils considèrent les savoirs comme produits de formations sociales ET discursives et soulignent l'importance à l'école des pratiques langagières et de leur spécificité disciplinaire malgré le poids de la forme scolaire (au sens de G. Vincent). Ces recherches s'appuient ainsi, pour les Sciences, sur les travaux de l'Histoire Sociale des Sciences, moins Latour (1988) que Pestre (1995) ou Licoppe (1996), montrant que toute (re)production 
de connaissances repose sur l'imbrication de technologies matérielles, discursives et sociales... et pour l'Histoire sur ceux de Détienne (1988), Ginzburg (2001), De Certeau (1975), Seixas (2000)... Cette attention portée aux Pratiques Sociales de Référence étendues au langage et dans les disciplines scolaires est articulée aux notions, empruntées à Bakhtine (1984), de "genres spécifiques aux sphères d'échanges », d' " ancrage social du signe », de " dialogisme », d' " hétéroglossie » (Marlot, Jaubert, Rebière, Doussot, Lhoste) qu'on peut mettre en relation avec le concept de polyphonie (Ducrot - Lhoste) ou de " polyphasie cognitive » (Moscovici - Souplet, thèse en cours). Toutes ces thèses d'une part convergent vers la nécessité de construire une "position énonciative » qui caractérise les savoirs en jeu et le contexte de leur usage et d'autre part évoquent la notion englobante de «communauté discursive disciplinaire » (Bernié, 2002, Jaubert, Rebière, Bernié, 2004). On constate d'ailleurs, dans les travaux les plus récents, un processus de banalisation des notions convoquées qui perdent leurs guillemets et ne sont plus interrogées. Certaines thèses ( $c f$. Marlot) articulent ce cadre théorique avec des concepts issus de la didactique des mathématiques «topo, chrono et méso genèse » et devenus outils de comparaison performants dans le cadre des recherches comparatistes (Mercier, SchubauerLeoni, Sensevy).

On peut remarquer que dans cette catégorie de recherches, les travaux menés par des chercheurs non linguistes ne développent (évidemment) pas de théorie du langage et la plupart s'appuient sur le cadre théorique ébauché par l'équipe de Bordeaux à partir des apports combinés de Bakhtine, Maingueneau, Grize, Vygotski, Brossard, Bronckart, François... diffusés chez les chercheurs en didactique du français, dont les travaux sont centrés sur la langue et/ou l'activité des élèves Bautier, Bucheton, Garcia-Debanc, Nonnon, Plane...

De fait, l'activité langagière disciplinaire peut être centrale, sans que soit développée une théorie du langage autre qu'une reprise rapide des concepts de « pratique langagière », " pratique sociale de référence », " communauté discursive disciplinaire scolaire » (Dupré, 2010). Par ailleurs des références à un linguiste comme Bakhtine ou aux travaux de Bernié, Jaubert, Rebière, (Bedouin, E, 2008) peuvent tenir lieu de cadrage théorique sur le plan langagier, alors que la théorie des mondes en tant que constructions culturelles empruntée à Popper ou la notion de communauté interprétative de Kuhn, sont convoquées, développées et auraient pu être articulées à la notion de «communauté discursive », comme le prévoit le programme actuel de recherches de l'équipe de Bordeaux.

Mais alors que les travaux fondateurs du champ reposent sur des fondements théoriques et heuristiques clairement définis, ( $c f$. les approches convergentes de Quilio et Sensevy sur la « pragmatique didactique » et de l'équipe de Bordeaux sur les boucles complexes de contextualisation - décontextualisation caractéristiques des Zones de Développement Proche), les usages qui vont en être faits ont pour simple point commun, de manière explicite ou non, le fait de considérer que le langage n'est pas transparent, qu'il est étroitement lié aux processus cognitifs et que son usage est toujours contextualisé. Tout se passe comme si le cadre théorique sur lequel repose la question du langage dans les disciplines était stabilisé, n'était plus à interroger et dès lors pouvait proposer un outillage pour analyser les énoncés contextualisés des élèves. Par exemple, dans toutes ces recherches, la notion de «position énonciative » ou « positionnement énonciatif» ou «pos- 
ture » ( $c f$. infra) est régulièrement pointée comme centrale sans pour autant faire l'objet d'une description approfondie : ainsi les trois désignations apparaissent interchangeables alors qu'on peut peut-être distinguer les deux premières sous l'angle résultat/processus et la troisième sous celui de sa composante sociale qui renvoie à l'habitus bourdieusien. Cette distinction supposerait un affinement de la notion et ouvrirait la question clé du travail de construction, de modification, de déplacement du sujet et de son rapport à l'activité. Par ailleurs, ces travaux ne permettent pas encore, à notre connaissance, d'établir la carte conceptuelle qui caractériserait les spécificités énonciatives de toutes les disciplines. En effet, même si plusieurs recherches, particulièrement dans le cadre de l'INRP (Douaire, 2004, Barré-De Miniac, Reuter, 2006.) présentent des analyses ancrées dans diverses disciplines, elles demeurent juxtaposées, au mieux comparées (Bédoin) et le travail « surplombant» amorcé par Plane et Garcia-Debanc (2003) qui pourrait proposer aux recherches en didactique du français une place au sein des démarches comparatistes est à poursuivre.

\subsection{Cadrage institutionnel}

Les recherches pour l'école ne peuvent faire l'impasse sur les textes institutionnels qui orientent les pratiques d'enseignement. Que prévoient les curriculums pour permettre à tous les élèves de construire les positions énonciatives pertinentes et de s'approprier les pratiques langagières attendues en chaque discipline?

Les textes officiels de 2002 se caractérisaient entre autres par l'importance accordée à la maîtrise des langages dans les différentes disciplines (cf. supra). En dehors de quelques incohérences entre les disciplines et d'une confusion regrettable en français entre les savoirs langagiers visés et les pratiques langagières pour les construire (de sorte que le langage n'est pas perçu comme outil de construction de savoirs en français, ce qui occulte toutes les activités de controverse sur le fonctionnement du langage, celles d'observation, de catégorisation et de stabilisation des savoirs sur la langue) ces textes signalaient, selon nous, une avancée importante dans la prise en compte du langage dans les disciplines. Cependant, ils ont pu être perçus comme une menace pour l'unité et la cohérence de la discipline (Hélène Romian, 2002, colloque de Québec, 2004).

Un balayage rapide des textes officiels de 2007 puis 2008 aurait pu dissiper leurs inquiétudes. En effet, l'accès à la maîtrise de la langue française y relève clairement de l'enseignement du français. En revanche, si les programmes des disciplines autres réfèrent très peu aux pratiques langagières, dans les progressions, certaines formulations antérieures sont maintenues, « décrire une figure, faire le récit de... »; enfin les paliers du socle sont eux rédigés en termes de « compétences » qui réfèrent nettement aux textes de 2002, « décrire, nommer, justifier...».

La rupture avec 2002 et avec la maîtrise de la langue au collège (1997) qui prônait une responsabilité partagée entre français et disciplines autres, est encore plus nette en ce qui concerne le collège : les pratiques langagières disciplinaires sont renvoyées dans les disciplines. De ce fait, les textes de français se focalisent sur les écrits littéraires à lire et à produire, les apprentissages langagiers 
se bornant à des apprentissages transversaux, « hors contenus » (par exemple «s'exprimer de manière appropriée »).

Si les textes de 2008 reconnaissent, même implicitement, l'importance de la maîtrise de la langue dans les apprentissages disciplinaires, le traitement de l'étude de la langue dans le cadre du français repose sur une conception représentationniste qui naturalise les savoirs, les empile sans prendre en compte les processus de catégorisation inhérents à la construction des concepts en jeu, les instrumentalise et exclut toute référence aux phénomènes énonciatifs : pas de définition des positions énonciatives disciplinaires, pas de comparaisons des usages de la langue, finalement pas d'ouvertures vers une éventuelle « secondarisation » (cf. infra) des pratiques langagières.

\section{Le positionnement énonciatif : un objet pour la didactique du français}

Lors des activités langagières mises en œuvre pour apprendre dans une discipline, les élèves se trouvent confrontés à des points de vue divergents et des modes de parler socialement différents. Il leur faut apprendre à construire la position énonciative propre à la discipline. Il en est de même dans toutes les disciplines. Nous faisons l'hypothèse que la mise en cohérence et la stabilisation de ces différentes positions relève de l'enseignement du français.

\subsection{Questions aux objets actuels de l'enseignement du français}

Les savoir faire ou compétences listés dans les textes officiels (décrire, raconter, argumenter, expliquer...) renvoient à des objets traditionnels de l'enseignement du français : les genres discursifs, enseignés en tant que formes (liste des caractéristiques discursives et linguistiques), hors contexte d'usage, et dont la maîtrise est le but, comme si la maîtrise de la forme permettait de comprendre l'activité. Or, nous pensons qu'aucun genre, pas plus qu'aucune notion grammaticale, hors de son contexte d'utilisation, n'est en soi un outil. Il le devient lorsqu'il aide l'élève à réguler son comportement de lecteur ou de producteur de textes : mais il ne peut y contribuer que s'il s'accompagne de ce que Piaget a appelé " schèmes d'utilisation » ${ }^{(6)}$ c'est-à-dire des sortes de plans d'action qui permettent de l'articuler avec des tâches ou problèmes à résoudre. D'où deux questions:

- Comment un schème d'utilisation peut-il être acquis hors d'un contexte d'utilisation? Comment comprendre ce que permet de faire un genre si l'on n'a pas imbriqué sa construction dans un faire? Non que le « discours sur », le discours

(6) Pour une utilisation didactique en direction de la notion de genre discursif, on se reportera à la contribution de Bernard Schneuwly, « Genres et types de discours : considérations psychologiques et ontogénétiques », in Les interactions lecture-écriture, Y. Reuter dir., Peter Lang, Berne, 1994. 
métalinguistique, n'ait aucune utilité, mais on peut s'interroger avec MarieLaure Elalouf ${ }^{(7)}$ sur ce qu'il permet d'appréhender de manière surplombante :

"Dans un contexte scolaire marqué par l'hétérogénéité sociale et culturelle, on ne peut réintroduire d'emblée des savoirs métalinguistiques décontextualisés sans fragiliser les élèves qui n'ont guère eu l'occasion de développer en dehors de l'école une attitude distanciée à l'égard de la langue ».

- Comment un outil peut-il aider un élève à réguler son comportement (fonction de ce que Vygotski appelle « l'outil psychologique ») s'il n'est pas subjectivement impliqué dans son appropriation, c'est-à-dire si l'extériorité de l'outil (déposé dans notre culture) ne se transforme pas en intériorité ? Comment un fait de langue peut-il devenir un outil ? La notion de « genres de l'activité » et le rapport qu'elle instaure entre activité, langage et subjectivité offre des pistes de réponse au champ didactique (Bernié, 2001), comme elle en a offert, dès la fin des années 80 , aux chercheurs hors didactique pour aborder les genres les plus transversaux du travail de l'école - prise de notes, résumés, comptes rendus ( $c f$. le titre provocateur de Grize : "Résumer mais pour quoi ? Résumer mais pour qui ? » $\left.{ }^{(8)}\right)$.

Ce qui est vrai pour les genres est vrai pour toutes les formes de la langue. Le langage pour construire les savoirs dans les disciplines peut-il alors s'enseigner hors de la discipline?

Le choix de travailler sur des conduites langagières contextualisées dans les disciplines scolaires signifie une prise de distance par rapport à deux modèles didactiques qui se chevauchent plus ou moins, sans grande cohérence :

- celui du « français scolaire», posé comme un objet clos sur lui-même (étudié en particulier par Josiane Boutet, 1999), et qui n'est qu'une variation (scolaire) dans les usages du langage ;

- celui du «français transversal », qui pose artificiellement une unification a priori des usages du langage autour de «types » éliminant la variation et donc toute la série des premières étapes de l'apprentissage (par exemple en posant l'existence d'un genre qui soit ne correspond à aucune des réalisations que peut rencontrer l'élève, soit donne l'exclusive à l'une d'entre elles en le désarmant dans toutes les situations où il aurait intérêt à s'appuyer sur une autre pour mener à bien telle activité cognitive). De plus, le langage du travail de l'école doit être assumé en tant que tel. Lui ajouter des finalités communicatives externes ne clarifie pas, selon nous, l'opacité des situations.

Ces deux modèles didactiques envisagent la cohérence discursive comme un objet formel traité en simple substitut de la cohésion. Les « mal dit », les ruptures, les dysfonctionnements sont généralement analysés en termes de manques et de difficultés linguistiques et non en termes d'homogénéité et d'adéquation à la finalité de la production. En effet ne peut-on interpréter ce phénomène dans

(7) IUFM de Versailles. Les lignes qui suivent proviennent d'un article du Français Aujourd'hui, $\mathrm{n}^{\circ} 135$, Octobre 2001, pp. 44-51.

(8) Actes du colloque de Pont-à-Mousson sur le résumé, L'activité résumante, M. Charolles et A. Petitjean dir., C.A.S. Université de Metz, 1992, coll. Didactique des textes. 
une perspective contextualiste ? emphase et thématisation inattendues, dénivellations ${ }^{(9)}$ hétérogènes, construction chaotique de l'objet disciplinaire... relèveraient alors du travail de positionnement énonciatif inhérent au processus d'apprentissage.

\subsection{Quelles approches du langage privilégier?}

Diverses recherches menées par les didacticiens ont fait émerger un certain nombre de concepts permettant de cerner un peu mieux ce qui se passe dans la classe et d'observer la manière dont les élèves s'emparent de l'espace discursif disciplinaire qui leur est proposé. Ces concepts articulés à une conception du langage toujours contextualisé et dialogique, pourraient proposer un cadre théorique heuristique aux recherches sur le langage dans les disciplines et à la question du positionnement énonciatif corollaire des apprentissages disciplinaires.

- Le concept d'activité. L'appropriation des savoirs est tributaire de l'activité de l'élève. Dans cette perspective, le langage sert au chercheur et à l'enseignant de fenêtre pour comprendre (un peu) l'activité du sujet et sa représentation du contexte. Observer l'activité langagière de l'élève devant une tâche c'est s'intéresser alors autant à ce qui est dit, qu'aux formes discursives (raconter, expliquer, commenter, définir, etc.), aux positionnements énonciatifs, aux marques de relations, conflits, dialogues internes, déplacements divers que le langage dessine. Ce point de vue suppose de ne pas considérer l'action humaine hors de son contexte et de prendre en compte ce que le sujet fait avec le langage, dans quel contexte il se situe et le degré de son adéquation avec le contexte visé par l'enseignant. Ainsi chaque discipline d'enseignement développe ses propres manières d'agir-penser-parler, en corrélation avec les savoirs visés. Si l'on se focalise sur l'aspect discursif, chaque classe engagée dans un apprentissage disciplinaire peut être considérée comme une «communauté discursive disciplinaire scolaire » (Maingueneau, 1984 ; Bernié, Jaubert et Rebière, 2004) qui représente le mouvement indispensable mais jamais achevé de construction des connaissances, à travers le traitement continu de la dimension langagière des contrats didactique et pédagogique.

- Les notions de «position(nement) énonciative(f)» (Maingueneau, 1993) et de « posture » (Bautier, 1992, 1995 ; Bucheton, 1999; Rebière, 2001) posent que tout discours est produit dans un champ de l'activité humaine qui a un effet sur l'énonciation elle-même. Le locuteur se projette dans une formation discursive spécifique et construit ainsi, au fil des discours, une position énonciative adaptée. Le positionnement énonciatif est donc fortement contextualisé. Cependant des schèmes d'activité langagière peuvent être partiellement automatisés, ce que désigne la «posture », d’origine sociologique (Bernstein), développée initialement par E. Bautier et D. Bucheton et qui pourrait trouver un développement didactique dans le cadre de la didactique comparée (Rebière). Alors que la circulation sur plusieurs positions énonciatives s'avère nécessaire aux apprentissages, la mise en œuvre systématique d'une posture due à une analyse erronée

(9) Reformulations énoncées d'un point de vue englobant ou au contraire plus spécifique (J.B. Grize, 1997). 
de 1'articulation action/ activité, et/ou relevant de pratiques sociales de référence en rupture avec les attentes scolaires pour un contexte donné, peuvent alors être source de malentendus et d'obstacle aux apprentissages.

- Dans cette optique, la notion de variation langagière, initialement centrée sur la variation sociologique, pourrait être repensée en termes de manières d'agir-penser-parler propres aux différents domaines de savoirs scolaires. Il s'agirait ainsi de travailler les processus d'inscription des élèves dans les univers disciplinaires scolaires pour aboutir, à terme, à des usages stabilisés permettant d'ancrer la construction d'une maitrise de la langue dans la confrontation des différentes pratiques langagières et discursives disciplinaires. Envisager la construction des connaissances métalinguistiques et des normes grammaticales suppose, comme balises intermédiaires du parcours, l'émergence d'objets d'enseignement nouveaux. Comme le texte intermédiaire dans le domaine de la production d'écrits n'est pas un "moindre texte » mais le lieu où s'élaborent des fonctionnements en voie de régulation (qui pourront se traduire peu à peu par des critères formalisés), la variation pourrait faire l'objet d'un traitement explicite, systématique et contextualisé. La notion de cohérence peut, dans ce cadre, être considérée comme signe de la maîtrise des paramètres contextuels de la production, qui permet l'adéquation du système d'énonciation à la visée communicative.

- La notion de «secondarisation » des discours réfère aux processus de transformation des usages langagiers initiaux des élèves, indissociable de la transformation de leurs modes d'agir et de penser dans une discipline. Elle cherche à rendre compte des mouvements de "saisie » (utilisation réfléchie) des outils culturels, via la construction de leurs schèmes d'utilisation. Le processus d'appropriation de ces outils génère des réorganisations ainsi que des transformations cognitives et langagières : réorganisation des systèmes lexicaux, des moyens discursifs du point de vue, des genres discursifs, etc. corrélés à des positionnements énonciatifs spécifiques, plus adéquats à la communauté discursive disciplinaire en voie d'institution dans la classe. La secondarisation est ainsi un outil d'analyse de l'inscription du sujet dans cette communauté. La mise à distance de ces processus modifie le rapport au langage et au monde de l'élève.

\section{En guise de conclusion : vers de nouveaux objets pour la didactique du français?}

Cependant la mise à distance ne peut guère se faire au cours des situations fonctionnelles, orientées par la construction des savoirs disciplinaires. Il semble donc nécessaire, en vue de construire des savoirs sur le langage, de travailler sur la matière langagière et la secondarisation des discours mis en œuvre dans les disciplines et de prévoir des situations de «mise à distance», de «structuration » et de comparaison des usages langagiers, dans le cadre de la classe de français. En effet, enseigner a priori des modèles phrastiques ou textuels à un élève ne suffit, selon nous pas pour qu'il perçoive ce qu'il peut faire avec une phrase ou comment un texte est un moyen d'agir pour apprendre et donc maîtriser le langage. Ce point de vue change le statut des « outils de la langue» dans l'apprentis- 
sage ainsi que l'articulation de leur acquisition avec l'acculturation, non seulement parce qu'aucune notion de grammaire ou de linguistique textuelle n'est en soi un outil mais parce qu'elle ne peut le devenir que si elle est une dimension de l'activité du sujet : une «faiblesse » langagière peut venir d'un problème de positionnement énonciatif.

Il nous semblerait donc pertinent d'identifier les spécificités langagières disciplinaires en matière de discours, ce qui implique aussi bien de prendre en compte des exigences de déplacement énonciatif des élèves dans des contextes variés que de construire un regard réflexif sur la langue. La classe de français semble être le milieu privilégié, comme l'étudient Plane et Garcia-Debanc à propos de l'argumentation, pour recenser, observer les divers discours produits dans le cadre des disciplines et prendre la mesure de leur dispersion, de la multiplicité des réalisations d'un même genre en fonction des disciplines ( $c f$. la description - Reuter, 2000 ; le récit, Pratiques, 133-134, 2007). Il serait ainsi possible d'aborder les phénomènes grammaticaux, les usages lexicaux, les figures, les stéréotypes et cadres de rationalité convoqués à partir des contraintes discursives, en lien avec le contexte de production et son contrat de communication. En revanche, en dehors de ce qui relève du code de base (un imparfait se conjugue toujours de la même manière, qu'il soit utilisé dans un contexte descriptif ou hypothético-déductif), les aspects communs de ces discours ne sont pas donnés d'emblée : ils sont à construire dans l'observation des différences, ce qui légitime une perspective variationnelle. Les poser a priori aboutit à des confusions qui masquent la spécificité des contextes ( $c f$. la polysémie lexicale ou la valeur de connecteurs comme donc, ensuite...).

Par ailleurs, il nous semble nécessaire d'étudier, en classe de français, non seulement les discours terminaux et stabilisés, mais aussi les discours «bricolés ", " fabriqués », intermédiaires qui correspondent aux phases de travail conceptuel. La comparaison permettrait de repérer le dialogisme des discours, les ancrages contextuels différents et leur caractère hétéroglossique (polyphonique), ce qui pourrait conduire à repenser l'enseignement de la cohérence et lui redonner sens, à partir des ruptures et des dysfonctionnements, signes de positions énonciatives fluctuantes et d'un ancrage contextuel non stabilisé. Il est possible en classe de français de comparer des listes de mots, de questions, d'enchaînements possibles ou non, nécessaires ou non, des processus de construction d'objet (Grize) dont les dénivellations (généralisation ou spécification) dans chaque discipline ( $c f$. la mare en littérature fantastique et en sciences; la distinction bébé/fœetus en sciences...) de façon à pointer les hiatus énonciatifs.

Enfin, (provisoirement) travailler la variation langagière permettrait d'objectiver les déplacements énonciatifs. Ainsi, la refiguration d'une même expérience en différentes disciplines permettrait, au-delà du recensement des objets dignes d'intérêt pour chacune, de chercher des moyens de les reformuler, d'ancrer le discours dans le contexte ad hoc, de mettre en œuvre les systèmes temporels... qui contribuent à clarifier le contexte d'échanges, à faciliter l'appropriation de savoirs sur la langue et à construire le point de vue surplombant et réflexif sur le langage.

Il nous semble que la notion de position énonciative est une question importante pour la didactique du français, en lien avec celle de conscience discipli- 
naire. Au croisement du psychologique, du social et du linguistique, elle ne peut se réduire à une question linguistique et être abordée pour elle-même en tant qu'objet. Parce qu'elle participe à la régulation des conduites langagières, son intégration dans l'enseignement du langage exige le développement de relations et de collaborations entre le français et les disciplines (y compris littérature et étude de la langue). Cette nécessité est soulignée, de manière récurrente, sous des formes différentes, à la fois dans les recherches et les textes officiels, sans que pour autant la reconfiguration de la discipline français n'ait vraiment pu la prendre en compte ${ }^{(10)}$.

\section{Bibliographie}

BAKHTINE, M. (1984) : Esthétique de la création verbale, Paris, Gallimard.

BARRÉ-DE MINIAC, C., REUTER,Y. (2006) : Apprendre à écrire au collège dans les différentes disciplines, Lyon, INRP.

BAUTIER, E. (1995) : Travailler en Banlieue, Business networks

- (1997) : "Pratiques langagières, activités des élèves et apprentissages », in la Lettre de la DFLM, $\mathrm{n}^{\circ} 21,10-13$.

- (1995) : Pratiques langagières, pratiques sociales, Paris, 1'Harmattan.

Bedouin, E. (2010) : «Croisements disciplinaires, enjeux didactiques : actualité d'une confrontation Littérature/Sciences », in Pratiques, n¹45-146, 161 178.

BERNIÉ, J.-P. (2001) : «Genres discursifs scolaires, genres de l'activité et conceptualisation ", in Bernié, J.-P. Apprentissage, développement et significations, Bordeaux, PUB, 155-171

— (2002) : «L'approche des pratiques langagières scolaires à travers la notion de "communauté discursive" : un apport à la didactique comparée ? » in Revue Française de Pédagogie, ${ }^{\circ} 141,77-88$.

BERNiE, J.-P., JAUBERT, M. et REBIERE, M. (2008) : « Du contexte à la construction du sujet cognitif : l'hypothèse énonciative », in Brossard, M. et Fijalkow, J. (Dir.) Vygotski et les recherches en éducation et en didactiques, Bordeaux, PUB, pp.123-141.

(10) Comme en témoigne l'absence de candidature pour le poste de professeur ouvert sur cette question, lors de la dernière campagne de recrutement des enseignants-chercheurs en université. 
Boutet, J. (2005) : " Pour une activité réflexive sur la langue », in Le Français Aujourd'hui, n¹48, AFEF,.65-74.

Bucheton, D. (1999) : «Les postures du lecteur », in P. Demougins et J.-F. Massol dir., Lecture privée, lecture scolaire : la question de la littérature à l'école, CRDP de Grenoble.

Bucheton, D., Chabanne, J.-C. (1998) : « Le point de vue, le doute et le savoir », in Le français aujourd'hui, $\mathrm{n}^{\circ} 123,16-29$.

- (2000) : «Conférence d'ouverture des journées d'étude de Perpignan (1999), L'écrit et l'oral réflexifs. Parler, écrire pour penser, apprendre et se construire », numéro spécial de la Lettre de la DFLM, 5-13.

Buty, C., Plantin, C. (dir) (2008) : Argumenter en classe de sciences, INRP.

CAILlier, J. (2005) : «Analyse du développement social, cognitif et langagier d'élèves en interaction dans des dispositifs de tutorat et débat entre pairs, et du point de vue des enseignants sur ces genres scolaires ", thèse soutenue à Montpellier 3, non éditée.

Charlot, B., BAUtier, E. RocheX, J.-Y. (1992) : École et savoir dans les banlieues... et ailleurs, Paris, Armand Colin.

Combettes, B. (2010) : Réflexion sur l'enseignement grammatical, in Pratiques, $\mathrm{n}^{\circ} 145-146,12-16$.

— (2008) : «Théories du changement et variations lnguistiques : la grammaticalisation », in Pratiques, $\mathrm{n}^{\circ} 137-139,135-145$.

DARNIS, F. (2003) : «Vers une didactique constructiviste en sports collectifs », in Amadescot Chantal, dir. Didactique de l'éducation physique, état des recherches, Paris, ed. Revue EPS, 79-102.

DAUnAY, B., REUTER, Y. (2008) : «La didactique du français : questions d'enjeux et de méthodes », in Pratiques, $\mathrm{n}^{\circ} 137-138,57-78$.

DECRON, A. (2007) : "Le récit éducatif, un tissage discursif complexe pour apprendre, penser et se construire », thèse soutenue à Montpellier 3, non éditée.

DOUAIRE, J. (dir) (2004) : Argumentation et disciplines scolaires, INRP.

ELALOUF, M.-L. (2001) : «Quand on rencontre la langue en travaillant sur le texte et le discours ", Le Français Aujourd'hui, n¹35. Paris, 44-51.

GARCIA-DEBANC, C. (1988) : «Propositions pour une didactique du texte explicatif », in Aster $n^{\circ}$ 6, 129-163.

- (1990a) : L'élève et la production d'écrits, CASUM, Metz, Presses Universitaires.

— (1990b) : «Didactique du français et didactique des disciplines scientifiques : convergences et spécificités ", in Perspectives didactiques en français, Actes du colloque de Cerisy 1979, coll. Didactique des textes, CASUM, Metz, Presses Universitaires.

— (1995) : «Une argumentation orale dans une démarche scientifique au cycle3 », in Repères $\mathrm{n}^{\circ} 12,79-103$.

- (1998) : " Interactions et construction des apprentissages dans le cadre d'une démarche scientifique ", in Repères, n¹7, 87-108.

GARCIA-DEBANC, C., Roger, C. (1986) : « Apprendre à rédiger des textes explicatifs », in Pratiques, $\mathrm{n}^{\circ}$ 51, 55-75. 
GRIZE, J. B. (1997) : Logique et langage, Paris, Ophrys.

HALTÉ, J.-F. (1998/2008) : «Le français entre rénovation et reconfiguration », in Pratiques 137-138, pp. 23-38.

Host, V. (1983) : Eveil scientifique et modes de communication, Paris, INRP.

JAUBERT, M. (2007) : Langage et construction des connaissances à l'école, Bordeaux, Presses universitaires de Bordeaux.

JAUBERT, M., REBIERE, M. (2001) : «Observer l'activité langagière des élèves en sciences », in Aster, n³0, pp. 173-196.

- (2002): «Pratiques de reformulations et construction de savoirs », in Aster, $\mathrm{n}^{\circ} 33$, pp. 81-110.

— (2002) : «Parler et débattre pour apprendre : comment caractériser un oral réflexif», in Chabanne, J.-C. et Bucheton, D.(dir.), Parler et écrire pour penser, apprendre et se construire, Paris, PUF, pp. 163-186.

JAUBert, M., REBIERE, M., BERnie, J.-P., (2004) : «L'hypothèse « communauté discursive », in Les cahiers Théodile 4, Univeristé Charles de Gaulle, Lille 3, pp. 51-80.

LeOntiEV, A. N. (fr.1984) : Le développement du psychisme, Paris, Editions sociales.

LETTRE DE LA DFLM (2000), $\mathrm{n}^{\circ} 26$.

MARCHAND, J.-C. 2002 : «Enseignement musical et jugement esthétique : leur intrication dans les pratiques évaluatives », in JREM vol.1 pp. 9-33.

Mercier, A., Schubauer-LeOni, M.-L., SenseVy, G. (2002) : « Vers une didactique comparée », » in Revue Française de Pédagogie, n¹41, pp. 5-16.

NonnOn, E. (1998) : «La notion de point de vue dans le discours », in Pratiques, $\mathrm{n}^{\circ} 100$.

- (2001): «La construction d'objets communs d'attention et de champs notionnels à travers l'activité partagée de description », in Grandaty M. et Turco G. (coord.) L'oral dans la classe : discours, méta-discours, interations verbales et construction de savoirs à l'école primaire, Paris, INRP.

Plane, S. et GARCIA-DEBANC, C. (2003) : " L'argumentation dans les disciplines : un objet qui intéresse la didactique du français », in actes sur CDRom du colloque Construction des connaissances et langage dans les disciplines d'enseignement, Bordeaux.

Pluvinage, F. 2000 : «Mathématiques et maîtrise de la langue », in Repère 39, pp. 115-126.

REBIERE, M. (2001) : «Une notion venue d'ailleurs... la posture », in J.-P. Bernié (dir.) Apprentissages, développement et significations, Bordeaux, PUB, 191-207.

REUTER, Y. (2007) : «La conscience disciplinaire, présentation d'un concept », in Education et didactique, 1,2, pp. 57-71.

- (2007) : «Penser les méthodes de recherche en didactique du français », in la Lettre de l'AIRDF, ${ }^{\circ} 40,5-8$.

- (2003) : «La représentation de la discipline ou la conscience disciplinaire ", in la Lettre de l'AIRDF, n³2, 18-22.

Romian, H. (2002) : «Le français au cycle 3 :chef d'œuvre en péril ? » in Former Des Maîtres 18, FSU. 
SCHNEUWLY, B. (2007) : «Le "français", une discipline scolaire autonome, ouverte et articulée », in Falardeau, E., Fisher, C., Simard, C., Sorin, N., La didactique du français, les voies actuelles de la recherche, Laval, Presses de 1’Université de Laval, 9-26.

SCHNEUWLY, B., DOLZ, J. (1997) : «Les genres scolaires, des pratiques langagières aux objets d'enseignements ", in Repères, ${ }^{\circ}$ 15, INRP, 27-40.

- (1997) : «Le concept d'activité. Quelques aspects qu'il rend visibles dans l'enseignement du français », in La Lettre de la DFLM, n²1, 6-10.

Vygotski, L. (fr. 1985), Pensée et langage, Paris, Editions Sociales.

\section{Colloques}

Construction des connaissances et langage dans les disciplines d'enseignement, Bordeaux (2004).

Faut-il parler pour apprendre (2004), Lille.

Le français, discipline singulière, plurielle ou transversale (2004), Québec.

Interactions didactiques (2010), Lyon.

\section{Documents officiels}

La maitrise de la langue à l'école (1992), MEN, CNDP.

La maitrise de la langue au collège (1997), MEN, CNDP.

Programmes pour l'école (2002-2008), MEN,CNDP.

\section{Thèses consultées}

BEDOIN, E. (2008) : Lire le texte, lire le monde : du jeu interprétatif en littérature et en sciences, Université de Rennes 2.

DARNIS, F. (2004) : Place et rôle du développement cognitif et de l'interaction sociale dans l'acte tactique en jeux et sports collectifs, Université de Bordeaux 2 .

DoussoT, S. (2009) : Ecrits non linéaires et apprentissage de l'histoire. Des pratiques langagières instrumentées par des listes et tableaux pour construire un savoir problématisé (collège, cycle 3). Université de Nantes.

DUPRE, A. (2010) : Lire en français et en sciences au cycle 3 : étude comparée dans six écoles de la métropole lilloise. Université de Lille.

Lhoste, Y. (2008) : Problématisation, activités langagières et apprentissages dans les sciences de la vie. Etude de débats scientifiques dans la classe dans deux domaines biologiques : nutrition et évolution. Université de Nantes.

MARLOT, C. (2008) : Caractérisation des transactions didactiques : deux études de cas en découverte du monde vivant au cycle 2, Université de Bretagne - Rennes 2.

SAID, F. (2010) : Débat scientifique en classe et acculturation scientifique chez des élèves français de seconde et des élèves tunisiens du secondaire, université de Bordeaux 2 et université virtuelle de Tunis. 